\title{
Diagonal Degree Correlations vs. Epidemic Threshold in Scale-Free Networks
}

\author{
M. L. Bertotti $(\mathbb{D})$ and G. Modanese $(D)$ \\ Free University of Bozen-Bolzano, Faculty of Science and Technology, I-39100, Bolzano, Italy \\ Correspondence should be addressed to M. L. Bertotti; marialetizia.bertotti@unibz.it
}

Received 26 July 2021; Revised 1 September 2021; Accepted 23 September 2021; Published 8 October 2021

Academic Editor: Xuzhen Zhu

Copyright (C) 2021 M. L. Bertotti and G. Modanese. This is an open access article distributed under the Creative Commons Attribution License, which permits unrestricted use, distribution, and reproduction in any medium, provided the original work is properly cited.

\begin{abstract}
We prove that the presence of a diagonal assortative degree correlation, even if small, has the effect of dramatically lowering the epidemic threshold of large scale-free networks. The correlation matrix considered is $P(h \mid k)=(1-r) P_{h k}^{U}+r \delta_{h k}$, where $P^{U}$ is uncorrelated and $r$ (the Newman assortativity coefficient) can be very small. The effect is uniform in the scale exponent $\gamma$ if the network size is measured by the largest degree $n$. We also prove that it is possible to construct, via the Porto-Weber method, correlation matrices which have the same $k_{n n}$ as the $P(h \mid k)$ above, but very different elements and spectra, and thus lead to different epidemic diffusion and threshold. Moreover, we study a subset of the admissible transformations of the form $P(h \mid k) \longrightarrow P(h \mid k)+\Phi(h, k)$ with $\Phi(h, k)$ depending on a parameter which leaves $k_{n n}$ invariant. Such transformations affect in general the epidemic threshold. We find, however, that this does not happen when they act between networks with constant $k_{n n}$, i.e., networks in which the average neighbor degree is independent from the degree itself (a wider class than that of strictly uncorrelated networks).
\end{abstract}

\section{Introduction}

From the mathematical point of view, a network is completely characterized (up to isomorphisms corresponding to simple redenominations of the vertices) when a list of links or an adjacency matrix is given. In many applications involving large networks, however, one often summarizes the information on the network structure in a statistic-probabilistic form, by introducing the two fundamental quantities $P(k)$ and $P(h \mid k) . P(k)$, called degree distribution, represents the probability that a randomly chosen vertex of the network has $k$ neighbors. $P(h \mid k)$, called degree correlation function, expresses the conditional probability that a vertex of degree $k$ is connected to a vertex of degree $h$. An alternative but equivalent description involves the symmetric quantities $e_{j k}$, defined as the probabilities that a randomly chosen link connects two vertices of degree $j$ and $k$ [1-3].

When one considers in a purely axiomatic way a class of networks, called Markovian networks [4], which are completely defined by assigning the quantities $P(k)$ and $P(h \mid k)$, one disregards higher-order correlations like $P(j|h| k)$, which can in general be present. It is interesting to investigate the connections between real networks and the corresponding Markovian networks. In the case of Barabasi-Albert networks, for instance, it is possible to use recipes for constructing ensembles of the two kinds (preferential attachment vs. rewiring) and compare them [5].

In any case, let us focus on $P(k)$ and $P(h \mid k)$. Imagine that we know them for a certain network and we want to study some dynamical processes on the network, e.g., epidemic diffusion processes [6]. It turns out $[7,8]$ that several features of these processes depend on a "contracted" form of the correlations, namely, the function $k_{n n}(k)$, called "average nearest neighbor degree" and defined as $k_{n n}(k)=\sum_{k=1}^{n} h P(h \mid k)$, where $n$ is the highest degree of the nodes of the network. This function of $k$ is simpler to analyse than the full matrix $P(h \mid k)$. Its increasing or decreasing character discriminates between assortative and disassortative networks (see [9-12] and refs.). We can further contract the information on the correlations into a single 
number, the Newman assortativity coefficient $r$, either using the $e_{j k}$ matrix or with one more summation procedure performed on $k_{n n}$.

One may wonder whether it is possible, given an admissible $k_{n n}$ function (it must satisfy a normalization condition given below), to compute a full correlation matrix which returns that $k_{n n}$ upon contraction on $h$. Porto and Weber have devised a method for this purpose [13], which has been used for some applications by themselves and Silva et al. [14]. However, while the correspondence $P(h \mid k) \longrightarrow k_{n n}(k)$ is univocal, this is not true for the opposite correspondence $k_{n n}(k) \longrightarrow P(h \mid k)$. One first scope of this work is to show explicitly this ambiguity in an important specific example, namely, that of a linear $k_{n n}$. To this end, we introduce in Section 2.1 the correlation matrix $P^{\mathrm{Vaz}-\mathrm{Wei}}(h \mid k)$ of Vazquez-Weigt [15], which has the simple form $(1-r) P_{h k}^{U}+r \delta_{h k}$, where $P^{U}$ is an uncorrelated matrix. The corresponding $k_{n n}$ is linear in $k$. Then, in Section 2.2, we recall the method by Porto and Weber for building a $P(h \mid k)$ starting from a $k_{n n}$, and in Section 2.3, we apply it to $k_{n n}$ of Vazquez and Weigt. A comparison of the result with the original matrix $P^{\mathrm{Vaz}-\mathrm{Wei}}(h \mid k)$ shows remarkable differences. Also, in Section 2.4, we briefly discuss the case of real networks for which the $k_{n n}$ function is only approximately linear.

While examining the differences noted in Section 2.3, we have been led to consider the eigenvalue spectra of the associated connectivity matrices $C_{k h}=k P(h \mid k)$. This has revealed a simple general property of the eigenvalues of $C^{\mathrm{Vaz}-\mathrm{Wei}}$, which has important consequences for the epidemic threshold in diffusion models based on this matrix (Section 3). In fact, the eigenvalues of $C^{\mathrm{Vaz}-\text { Wei }}$ are $\Lambda^{(i)}=r i$, where $i=1, \ldots, n$ and $n$ is the largest degree in the network. It follows that the epidemic threshold $\lambda_{c}=1 / \Lambda^{\max }$ (Section 3.1) is proportional to $n^{-1}$, for any fixed value of $r$. When $r$ is small, the epidemic threshold $\lambda_{c}$ is definitely greater than zero for small networks, but if $n \longrightarrow \infty$, the threshold goes quickly to zero. The convergence is much faster than for other correlations, for which the largest eigenvalue typically grows as a root of $n$ or even as $\ln n$ when the scale-free exponent $\gamma$ is equal to 3 (Sections 3.2 and 3.3). The conclusion is that adding even a very small amount of assortative diagonal degree correlations to an uncorrelated network leads, in the large- $n$ limit, to a fast vanishing of the epidemic threshold. Further, numerical results for the case in which the assortativity coefficient is not small are given in Section 3.4 .

In Section 4, we discuss a family of transformations of the correlation matrices which keep their $k_{n n}$ functions unchanged. We show that such transformations affect in general the epidemic threshold even if this does not happen when these transformations act on networks in which the average neighbor degree is independent from the degree itself; in this case, the transformations lead to networks for which the epidemic threshold remains unchanged and which, albeit having a constant $k_{n n}$, belong to a wider class than that of strictly uncorrelated networks.

\section{The Correlation Matrix of Vazquez-Weigt vs. That of Porto-Weber Reconstruction}

2.1. The Vazquez-Weigt Matrix. Vazquez and Weigt [15] have defined the following assortative correlation matrix:

$$
P^{\mathrm{Vaz}-\mathrm{Wei}}(h \mid k)=(1-r) \frac{h P(h)}{\langle k\rangle}+r \delta_{h k},
$$

where $\langle k\rangle=\sum_{k=1}^{n} k P(k)$ (more generally, below, $\langle g(k)\rangle$ denotes $\sum_{k=1}^{n} g(k) P(k)$ for any function $g$ ).

This Ansatz has been used in several applications [16]. It is a linear combination of a perfectly uncorrelated matrix with elements $h P(h) /\langle k\rangle$, giving a probability of connection independent from $k$ and a perfectly assortative matrix $\delta_{h k}$ (giving a nonzero probability of connection only between nodes of the same degree). The coefficient $r$ in the linear combination can vary in the range $[0,1]$ and corresponds to the Newman assortativity coefficient.

The $k_{n n}$ function for the Vazquez-Weigt correlation matrix is easily found as follows:

$$
k_{n n}^{\mathrm{Vaz}-\mathrm{Wei}}(k)=\sum_{k=1}^{n} h P^{\mathrm{Vaz}-\mathrm{Wei}}(h \mid k)=(1-r) \frac{\left\langle k^{2}\right\rangle}{\langle k\rangle}+r k .
$$

Since the first term is independent from $k$, this is a linear function, with slope $r$.

2.2. The Recipe of Porto-Weber for a Correlation Matrix Having a Predefined $k_{n n}$. In order to compute the correlation matrix $P(h \mid k)$ starting from a given function $k_{n n}$ (normalized as $\left.\sum_{k} k P(k) k_{n n}(k)=\left\langle k^{2}\right\rangle[12]\right)$, Porto and Weber define first the symmetric function as follows:

$$
f(h, k)=1+\frac{\left[k_{n n}(h)-k_{m e}\right]\left[k_{n n}(k)-k_{m e}\right]}{\left\langle k k_{n n}\right\rangle_{e}-k_{m e}^{2}},
$$

where

$$
\begin{aligned}
k_{m e} & =\frac{\left\langle k^{2}\right\rangle}{\langle k\rangle}, \\
\left\langle k k_{n n}\right\rangle_{e} & =\sum_{h} \frac{h P(h)}{\langle k\rangle} h k_{n n}(h) .
\end{aligned}
$$

In other words, $\left\langle k k_{n n}\right\rangle_{e}$ is the average of the quantity $k k_{n n}(k)$ with a normalized "edge" probability distribution defined as $P_{e}(k)=k P(k) /\langle k\rangle$, giving the probability that a randomly chosen edge of the network is connected to a node of degree $k$.

The conditional probability $P(h \mid k)$ is then given by

$$
P(h \mid k)=\frac{h P(h)}{\langle k\rangle} f(h, k) .
$$

It is immediate to check that $P(h \mid k)$ defined in this way satisfies the normalization condition in $h$ and the network closure condition as follows: 


$$
h P(k \mid h) P(h)=k P(h \mid k) P(k), \quad \text { for all } h, k=i=1, \ldots, n .
$$

Also, it is straightforward to replace $P(h \mid k)$ into the definition of $k_{n n}(k)$ and obtain an identity.

2.3. Porto-Weber Recipe Applied to the Vazquez Correlation Matrix. Now, suppose we want to reconstruct $P^{\mathrm{Vaz}-\mathrm{Wei}}(h \mid k)$ starting from $k_{n n}^{\mathrm{Vaz}-\mathrm{Wei}}(k)$ using the Porto-Weber recipe. Applying this recipe to the $k_{n n}$ function of Vazquez-Weigt, one obtains

$$
f(h, k)=1+r \frac{\left(h-k_{m e}\right)\left(k-k_{m e}\right)}{\left\langle k^{3}\right\rangle /\langle k\rangle-k_{m e}^{2}} .
$$

One can check numerically that the insertion of this function $f(h, k)$ into the Porto-Weber recipe gives a correlation matrix $P^{\text {Por-Web }}(h \mid k)$ whose $k_{n n}$ coincides element by element with $k_{n n}$ of Vazquez-Weigt. However, $P^{\text {Por-Web }}(h \mid k)$ does not coincide with $P^{\mathrm{Vaz}-\mathrm{Wei}}(h \mid k)$. The difference between the two matrices is evident looking at their dependence on $h$ and $k$. Their traces and eigenvalues are markedly different as we shall show in the next section. A graphical representation showing the differences of the single elements is given in Figures 1 and 2.

In conclusion, with the Porto-Weber recipe, it is possible to obtain from a given $k_{n n}(k)$ function a correlation matrix $P(h \mid k)$ which yields that $k_{n n}$, but such a correlation matrix is not the unique correlation matrix having the given $k_{n n}$ as its average nearest neighbor degree function. This could and should in fact be expected since the definition of $k_{n n}$ involves a summation, and thus any two matrices $P^{(1)}(h \mid k)$ and $P^{(2)}(h \mid k)$, suitably normalized, such that

$$
\sum_{h=1}^{n} h\left[P^{(1)}(h \mid k)-P^{(2)}(h \mid k)\right]=0,
$$

yield the same $k_{n n}$.

We also observe that there is no guarantee that the Porto-Weber method works for any normalized $k_{n n}$. For instance, for a linear $k_{n n}$, some (unacceptable) negative values of $P^{\text {Por-Web }}(h \mid k)$ are obtained when $r$ is greater than a value which is approximately 0.5 . For the $k_{n n}$ functions of Reference [14], of the form $k_{n n}=c k^{\alpha}$, one obtains negative values of $P^{\text {Por-Web }}(h \mid k)$ when $\alpha$ is greater than a value which is approximately 0.4 .

2.4. The Case of Real Networks. Summarizing, from the purely mathematical point of view, our argument above shows explicitly that fixing a $k_{n n}$ function of a network is not sufficient to define its full correlations. Actually, as proven in Section 4, there exists in general an infinite set of transformations of the correlations $P(h \mid k)$ which leave their $k_{n n}$ invariant. One can say that $P^{\mathrm{Vaz}-\mathrm{Wei}}$ and $P^{\text {Por-Web }}$ just represent mathematically two different Markovian networks (also having different connectivity spectra, as discussed in Section 3).

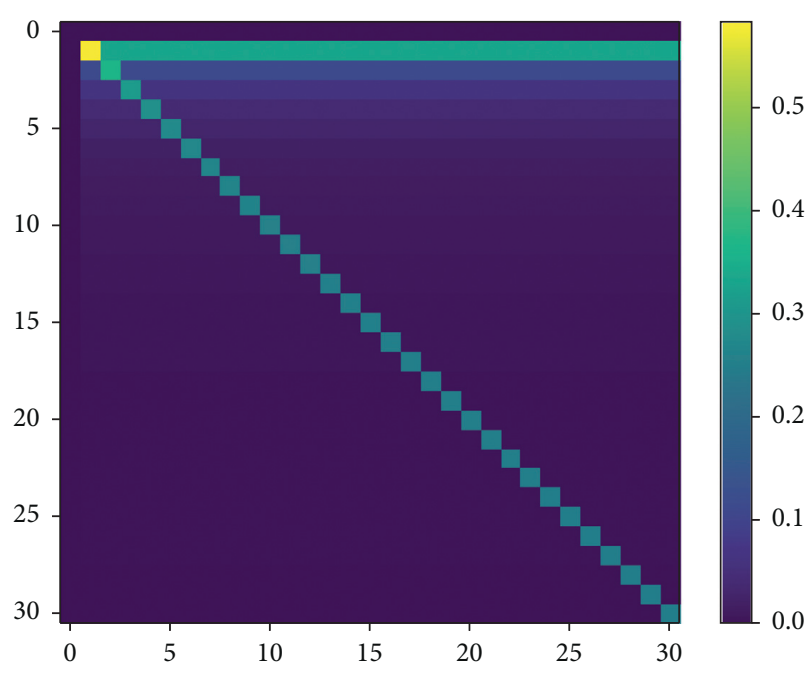

Figure 1: The correlation matrix $P^{\mathrm{Vaz}-\mathrm{Wei}}(h \mid k)$ with $n=30$ (maximum degree), $r=0.25$ (Newman assortativity coefficient), $\gamma=2.5$ (scale-free exponent). The diagonal elements are clearly visible. Like for any correlation matrix, each column is normalized to 1 (please note that row 0 and column 0 appear in the plot but do not actually belong to the matrix).

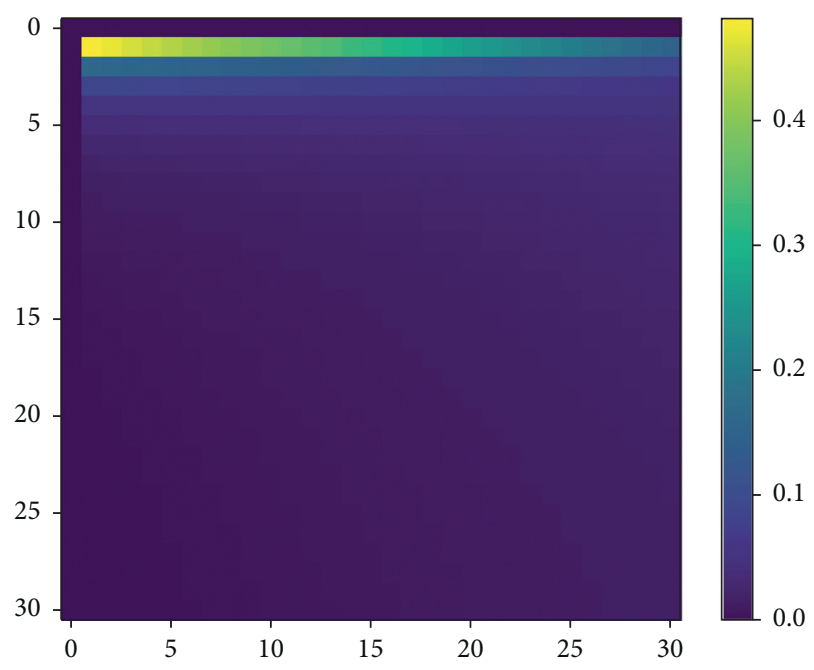

Figure 2: The correlation matrix $P^{\text {Por-Web }}(h \mid k)$ with $n=30$, $r=0.25$, and $\gamma=2.5$. Note the difference with $P^{\mathrm{Vaz}-\mathrm{Wei}}(h \mid k)$ in Figure 1 in spite of the fact that the two matrices have the same (linear) $k_{n n}$ function. In the "deep blue" lower part of the matrix, there are variations which are not visible in this plot but are obviously needed to keep each column normalized to 1 . The example is compared in Figure 3.

However, when it comes to the modelization of diffusion on real networks, the following question may arise: if we know that a certain real network displays a linear $k_{n n}$ function, is it more appropriate to compute its diffusion properties, and in particular the epidemic threshold, using a full correlation matrix of the $P^{\mathrm{Vaz}-\mathrm{Wei}}$ type or the $P^{\mathrm{Por}-\mathrm{Web}}$ type? This question is motivated by the fact that "empirical" $k_{n n}$ functions are often used to summarize in an efficient way the information available on correlations in real networks 


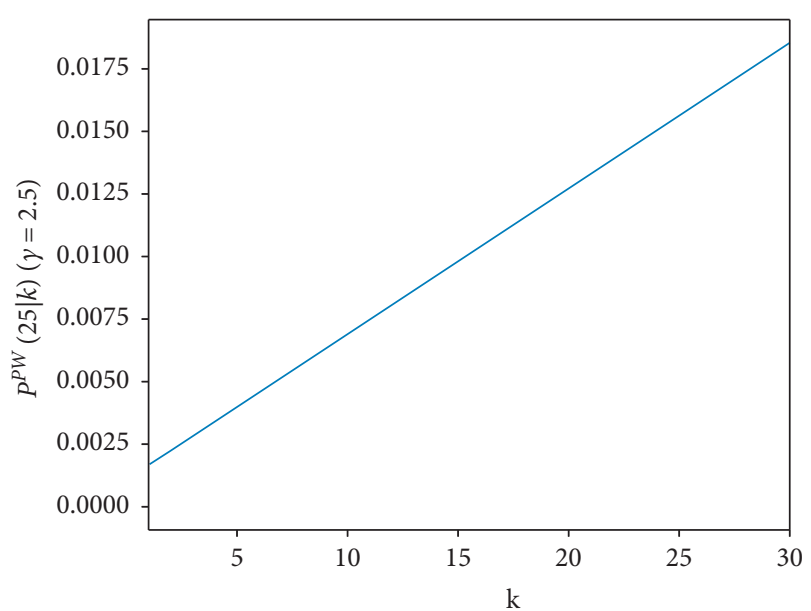

Figure 3: The elements of row 25 of the matrix $P^{\text {Por-Web }}$ of Figure 2. There is an increase in $k$ which is not visible in the color scale of Figure 2 but is necessary to preserve the normalization of the columns. The same happens of course for the other rows.

[3]. It is also known that a linear behavior of $k_{n n}$ for real assortative networks is quite frequent but only approximate. Especially for large values of the node degree $k$, the linear behavior is not maintained in practice, and one observes instead a decreasing tail in $k_{n n}$. This happens also because in real scale-free networks, the largest hubs are present but rare, and the Markovian-probabilistic approach is stretched to its limits of applicability.

It can be shown through numerical simulations based on degree-preserving rewiring [12] that in a real assortative scale-free networks (even with high $r$ ), the large hubs cannot be connected to a sufficient number of other hubs so as to keep $k_{n n}$ increasing for large $k$ simply because many hubs have in principle a nonzero probability but are missing in a random concrete realization of the network. In such cases, using the Porto-Weber method to construct the full correlations starting from a realistic $k_{n n}$ function is clearly the more correct recipe.

\section{Differences in the Spectrum of the Connectivity Matrix}

3.1. The Connectivity Matrix and Its Relation with the Epidemic Threshold. For a Markovian network with correlation matrix $P(h \mid k)$, the associated "connectivity matrix" is defined as follows $[7,8,14]$ :

$$
C_{k h}=k P(h \mid k) \text {. }
$$

This matrix plays an important role in studies of diffusion on networks. For instance, in the Homogeneous Mean Field approximation of the SI (Susceptible-Infected) epidemic model, the equation set which describes the behavior in time of the fraction $\rho_{k}$ of infected nodes with degree $k$ is as follows (see [8]):

$$
\frac{\mathrm{d} \rho_{k}}{\mathrm{~d} t}=-\rho_{k}+\left(1-\rho_{k}\right) \lambda \sum_{h=1}^{n} k P(h \mid k) \rho_{h}, \quad k=1, \ldots, n .
$$

It can be generally shown that the solutions of this equation set are characterized by an "epidemic threshold" $\lambda_{c}$ which separates different spreading scenarios: if $\lambda>\lambda_{c}$, the system reaches a stationary state with a finite fraction of infected population, while if $\lambda<\lambda_{c}$, the contagion dies out exponentially fast. The threshold $\lambda_{c}$ turns out to be equal to $1 / \Lambda^{\max }$, where $\Lambda^{\max }$ is the largest eigenvalue of the connectivity matrix $C$.

3.2. The Epidemic Threshold for Uncorrelated Scale-Free Networks. It is therefore important to know the largest eigenvalue of the $C$ matrix, and a general result valid for scale-free networks $[7,8]$ states that this eigenvalue tends to $+\infty$ when the size of the network grows. This means that for large scale-free networks, the epidemic threshold is essentially zero and the epidemics spreads and persists in the population also when the contagion probability is very small.

In the absence of degree correlations (i.e., with uncorrelated $C$, namely, $\left.C_{k h}^{U}=k P^{U}(h \mid k)=k h P(h) /\langle k\rangle\right)$, it has been shown that

$$
\Lambda^{\max }=\frac{\left\langle k^{2}\right\rangle}{\langle k\rangle} .
$$

For scale-free networks with scale exponent $2<\gamma<3$, $\langle k\rangle$ is finite when the maximum degree $n$ tends to infinity, while $\left\langle k^{2}\right\rangle$ is divergent:

$$
\left\langle k^{2}\right\rangle \simeq \int_{k_{\min }}^{n} \frac{c_{\gamma}}{k^{\gamma}} k^{2} \mathrm{~d} k=\left[\frac{k^{3-\gamma}}{3-\gamma}\right]_{k_{\min }}^{n},
$$

where $c_{\gamma}$ is the normalization constant of the degree distribution $P(k)=c_{\gamma} / k^{\gamma}$. The divergent part of (12) is of the form $n^{3-\gamma}$; thus, $\lim _{n \rightarrow \infty} \Lambda^{\max }=+\infty$ for $\gamma \in[2,3)$. When $\gamma=3$, the limit is also infinite but only with slow divergence $\sim \ln n$.

3.3. The Epidemic Threshold for the Assortative Networks of Vazquez-Weigt. It can be shown through general arguments that the divergence of the largest eigenvalue $\Lambda^{\max }$ when $n \longrightarrow \infty$ holds true independently from the degree correlations (see [4, 7]; see also some special cases in [12]). However, in the case of the diagonal assortative correlation matrices introduced by Vazquez and Weigt, a simple direct proof is possible, which is not yet available in the literature. Since for these correlations the assortativity level (expressed through the Newman coefficient $r$ ) is easily tunable in the range $[0,1]$, the proof also has interesting consequences for the epidemic threshold in general.

The connectivity matrix associated to $P^{\mathrm{Vaz}-\mathrm{Wei}}(h \mid k)$ is

$$
C_{k h}^{\mathrm{Vaz}-\mathrm{Wei}}=(1-r) \frac{k h P(h)}{\langle k\rangle}+r k \delta_{k h} .
$$

In order to compute its eigenvalues $\Lambda^{(i)}$, we consider the determinant of the matrix $(C-\Lambda I)$, with elements

$$
C_{k h}^{\mathrm{Vaz}-\mathrm{Wei}}-\Lambda \delta_{k h}=(1-r) \frac{k h P(h)}{\langle k\rangle}+(r k-\Lambda) \delta_{k h} .
$$




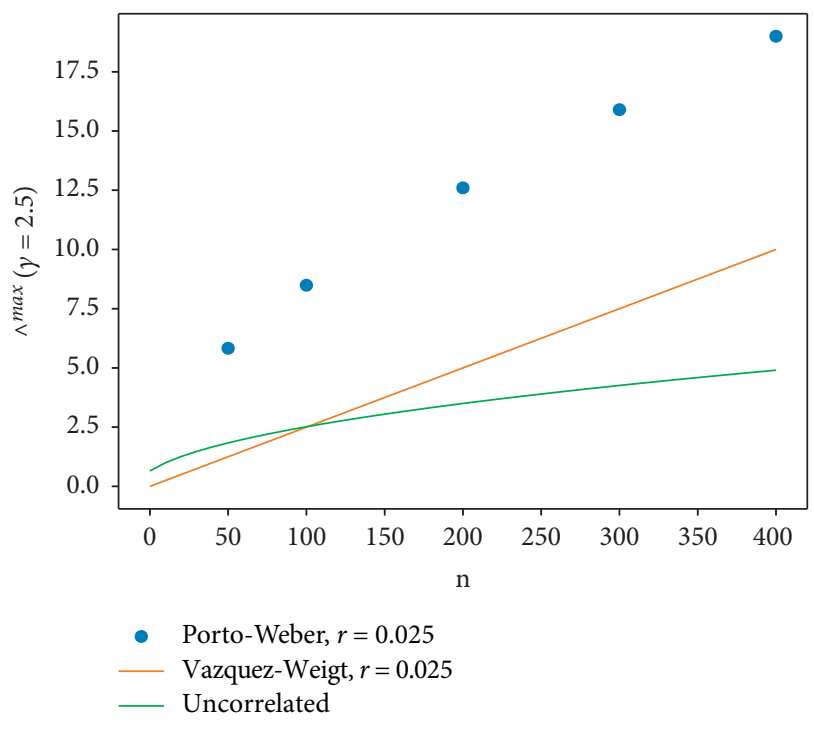

Figure 4: Behavior, as a function of the maximum degree $n$, of the largest eigenvalue $\Lambda^{\max }$ of the connectivity matrix, with scale-free exponent $\gamma=2.5$. The two continuous lines represent the case of uncorrelated networks and networks with diagonal correlations of the Vazquez-Weigt kind and assortativity coefficient $r=0.025$. The dots represent the case of networks reconstructed with the Porto-Weber recipe from the $k_{n n}$ function of the Vazquez-Weigt networks. Note that when $n$ increases, the largest eigenvalue increases faster (linearly) for the Vazquez--Weigt networks than for uncorrelated networks even though the assortativity is very small. Also, for the Porto-Weber networks, the increase is faster than for uncorrelated networks, but it is less than linear, and this gets worse when $\gamma$ increases (compare Figures 5 and 6).

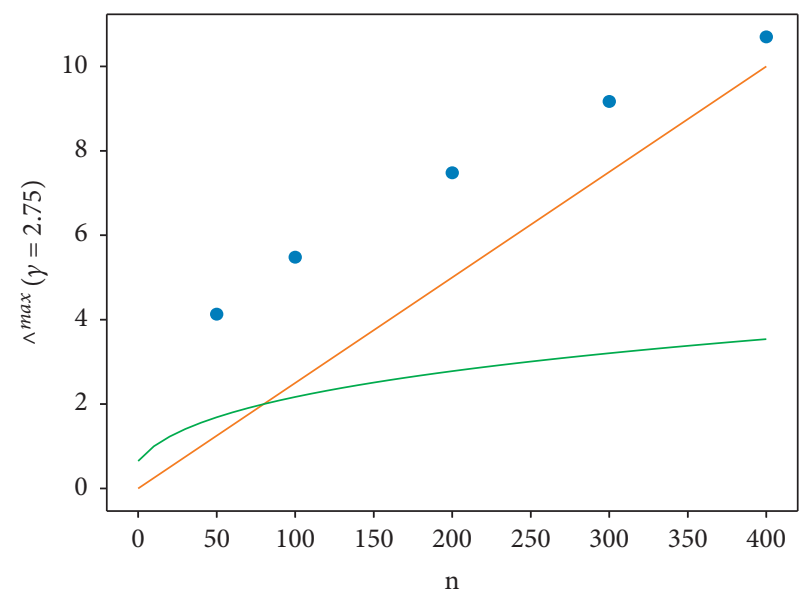

Porto-Weber, $r=0.025$
- Vazquez-Weigt, $r=0.025$
Uncorrelated

Figure 5: Behavior, as a function of the maximum degree $n$, of the largest eigenvalue $\Lambda^{\text {max }}$ of the connectivity matrix, with scale-free exponent $\gamma=2.75$. The case of $\gamma=2.5$ is compared in Figure 4 .

It is immediate to note that when $(r k-\Lambda)=0$, the determinant of this matrix is zero because the matrix $k h P(h)$ has rank 1 . Thus, we immediately find the $n$ eigenvalues as follows:

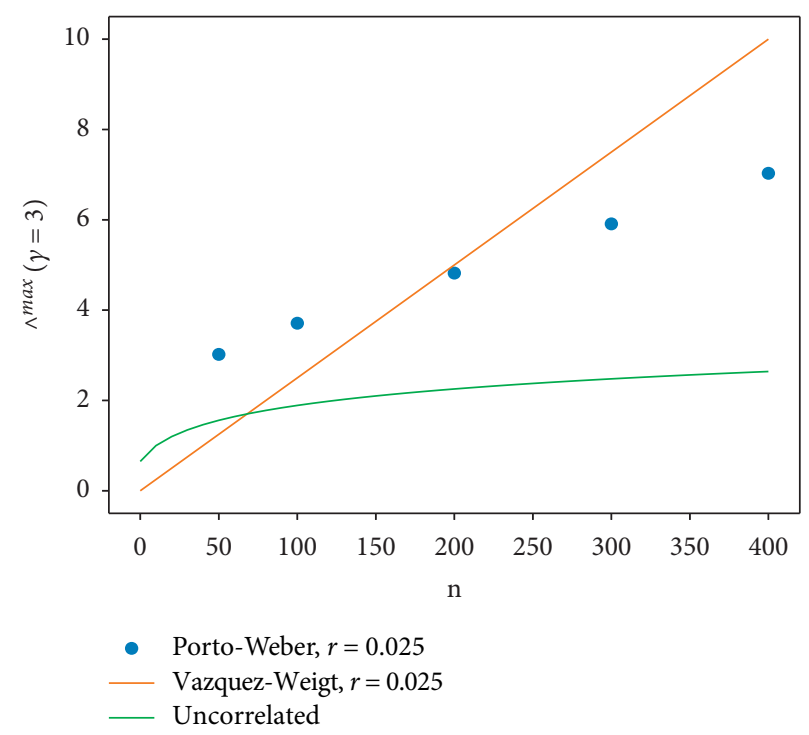

Figure 6: Behavior, as a function of the maximum degree $n$, of the largest eigenvalue $\Lambda^{\max }$ of the connectivity matrix, with scale-free exponent $\gamma=3$. The cases of $\gamma=2.5$ in Figure 4 and $\gamma=2.75$ in Figure 5 are compared.

$$
\Lambda^{(i)}=r i, \quad i=1, \ldots, n,
$$

the largest of them being $\Lambda^{\max }=r n$. When $n \longrightarrow \infty$, this eigenvalue grows much faster than the largest eigenvalue for uncorrelated networks, especially if $\gamma$ approaches 3 . For large networks, even a small value of $r$ like $r=0.025$ is sufficient for this to occur (see Figures 4-6).

Reference [17] reports the results of numerical simulations which, in retrospect, can be understood as being referred to a similar case of small $r$. On the analytical side, however, no general treatment was given but only an approximated eigenvalue expansion valid for $r$ close to 1 .

The conclusion is that for large scale-free networks, the presence of a small diagonal assortative correlation guarantees a quick convergence to zero of the epidemic threshold. We recall that according to the criterion by Dorogovtsev and Mendez [18], the relation between network size $N$ (number of nodes) and maximum degree $n$ is $N \sim n^{\gamma-1}$. Therefore, the behavior of $\Lambda^{\max }$ is insensitive to $\gamma$ as a function of $n$ but not of $N$. Still, even for $\gamma=3$, the dependence of $n$ on $N$ is $n \sim \sqrt{N}$, which is fast compared with the very slow increase in $\Lambda^{\max }$ in an uncorrelated network $\left(\Lambda^{\max } \sim \ln N\right)$.

3.4. Larger Values of $r$. In the mathematical diffusion theory on scale-free networks, it is interesting to consider the correlation matrix $P^{\mathrm{Vaz}-\mathrm{Wei}}$ in the limit of very small $r$ because this shows the existence of networks which are practically uncorrelated but have an epidemic threshold converging to zero much more quickly than for purely uncorrelated networks. From the point of view of the "statistical mechanics of networks," the set of these almost uncorrelated networks (say, with $0<r<\varepsilon$, and $\varepsilon$ small) is wider than the set of networks with $r=0$ exactly and can 
therefore be relevant in evaluations over statistical ensembles.

On the other hand, as discussed in Section 2.4, the alternative correlation matrix $P^{\text {Por-Web }}$ can improve the modeling of diffusion on real networks which display a $k_{n n}$ function that is only approximately linear, especially at large $k$. For this purpose, it is also interesting to compare the largest eigenvalues of $P^{\mathrm{Vaz}-\mathrm{Wei}}$ and $P^{\text {Por-Web }}$ for greater values of the assortativity coefficient $r$. Some results in this direction are reported in Table 1 . The general trend is confirmed as follows: $\Lambda^{\max }$ grows more quickly for $P^{\mathrm{Vaz}-\mathrm{Wei}}$.

\section{Examples of Variations of a Matrix $P(h \mid k)$ Which Do Not Modify Its Average Nearest Neighbor Degree Function $k_{n n}$}

In this section, we explore the more general question relative to multiplicity and concrete construction of variations of a correlation matrix $P(h \mid k)$ which do not modify the average nearest neighbor degree function $k_{n n}$. If such a variation is represented as

$$
P(h \mid k) \longrightarrow P(h \mid k)+\Phi(h, k)
$$

then the elements $\Phi(h, k)$ with $h, k=1, \ldots, n$ are required to

(1) Satisfy the Network Closure Condition (in this case, also the elements $P(h \mid k)+\Phi(h, k)$ do it as one can easily check);

(2) Satisfy the normalization according to which $\sum_{h}(P(h \mid k)+\Phi(h, k))=1$ (for each $k$, the elements $P(h \mid k)+\Phi(h, k)$ give the probabilities that a vertex with degree $k$ is connected to a vertex with degree $h$ ), which becomes in this case

$$
\sum_{h} \Phi(h, k)=0, \quad k=1, \ldots, n
$$

(3) Leave $k_{n n}$ unchanged which only occurs when

$$
\sum_{h} h \Phi(h, k)=0, \quad k=1, \ldots, n
$$

holds true.

In addition, the inequalities

$$
P(h \mid k)+\Phi(h, k) \geq 0, \quad \text { for all } h, k=1, \ldots, n,
$$

TABLE 1: Dependence of the largest eigenvalue of the connectivity matrix on the dimension $n$ (maximum degree) for some networks with assortativity index $r$ not close to zero.

\begin{tabular}{lccc}
\hline$n$ & $\Lambda_{U}^{\max }$ & $\Lambda_{P W[V W]}^{\max }(r=0.1)$ & $\Lambda_{P W[V W]}^{\max }(r=0.2)$ \\
\hline 100 & 4.80 & $8.59[10]$ & $13.8[20]$ \\
200 & 5.99 & $14.5[20]$ & $25.5[40]$ \\
300 & 6.80 & $20.4[30]$ & $36.9[60]$ \\
400 & 7.42 & $26.1[40]$ & $48.3[80]$ \\
\hline
\end{tabular}

The networks considered in this table have scale-free exponent $\gamma=2.75$ and thus correspond to those of Figure 5 but with a larger $r$ (for networks with exponents 2.5 and 3 , the behavior is similar). The second column gives the maximum eigenvalue for an uncorrelated network, as reference. The third column gives the maximum eigenvalue computed numerically for Porto-Weber networks with $r=0.1$ reconstructed from a linear $k_{n n}$; in each bracket is reported the corresponding largest eigenvalue for the Vazquez-Weigt network with the same $k_{n n}$, which is exactly equal to $r n$. The fourth column gives the eigenvalues for $r=0.2$. For larger values of $r$, e.g., $r=0.3$, the Porto-Weber recipe cannot be applied since it produces some negative matrix elements. In general, one observes, like in the case of very small assortativity, that the fastest increase in the eigenvalues occurs with the Vazquez-Weigt networks.

must hold true.

To obtain condition (1), we start and assume, on the trail of Porto and Weber,

$$
\Phi(h, k)=\frac{h p_{h}}{\langle k\rangle} \phi_{h, k},
$$

where $\phi_{h, k}$ is a symmetric matrix. Notice that from now on we will write $p_{h}=P(h)$ for the sake of brevity. Moreover, we will assume that each $p_{h}$ (for $h=1, \ldots, n$ ) is different from zero. The two conditions (2) and (3) then take the form

$$
\left\{\begin{array}{l}
\sum_{h} h p_{h} \phi_{h, k}=0 \text { for all } k=1, \ldots, n \\
\sum_{h} h^{2} p_{h} \phi_{h, k}=0 \text { for all } k=1, \ldots, n .
\end{array}\right.
$$

We will first look for matrices $\phi=\left\{\phi_{h, k}\right\}$ which satisfy (9) and only afterwards, in connection with some specific $P(h \mid k)$, will we check and specify when inequality (9) is satisfied too.

We narrow our search to the family of $n \times n$ symmetric matrices $\phi$, whose only nonzero elements are, together with $\phi_{1, n}$ and $\phi_{n, 1}$, those on the main diagonal and those on the first diagonal below and on the first diagonal above the main diagonal: 


$$
\phi=\left[\begin{array}{ccccccccccc}
\phi_{1,1} & \phi_{1,2} & 0 & 0 & 0 & \ldots & 0 & 0 & 0 & 0 & \phi_{1, n} \\
\phi_{1,2} & \phi_{2,2} & \phi_{2,3} & 0 & 0 & \ldots & 0 & 0 & 0 & 0 & 0 \\
0 & \phi_{2,3} & \phi_{3,3} & \phi_{3,4} & 0 & \ldots & 0 & 0 & 0 & 0 & 0 \\
\ldots & \ldots & \ldots & \ldots & \ldots & \ldots & \ldots & \ldots & \ldots & \ldots & \ldots \\
0 & 0 & 0 & 0 & 0 & \ldots & 0 & 0 & \phi_{n-1, n-2} & \phi_{n-1, n-1} & \phi_{n-1, n} \\
\phi_{1, n} & 0 & 0 & 0 & 0 & \ldots & 0 & 0 & 0 & \phi_{n-1, n} & \phi_{n, n}
\end{array}\right] .
$$

For any such matrix $\phi$, solving system (9) amounts to solve a linear system of $2 n$ equations in $2 n$ variables (recall that $\phi$ is symmetric):

$$
\left\{\begin{array}{l}
p_{1} \phi_{1,1}+2 p_{2} \phi_{1,2}+n p_{n} \phi_{1, n}=0 \\
p_{1} \phi_{1,1}+2 p_{2} \phi_{2,2}+3 p_{3} \phi_{2,3}=0 \\
2 p_{2} \phi_{2,3}+3 p_{3} \phi_{3,3}+4 p_{4} \phi_{3,4}=0 \\
\ldots \ldots \\
(n-2) p_{n-2} \phi_{n-2, n-1}+(n-1) p_{n-1} \phi_{n-1, n-1}+n p_{n} \phi_{n-1, n}=0 \\
p_{1} \phi_{1, n}+(n-1) p_{n-1} \phi_{n-1, n}+n p_{n} \phi_{n, n}=0 \\
p_{1} \phi_{1,1}+4 p_{2} \phi_{1,2}+n^{2} p_{n} \phi_{1, n}=0 \\
p_{1} \phi_{1,1}+4 p_{2} \phi_{2,2}+9 p_{3} \phi_{2,3}=0 \\
4 p_{2} \phi_{2,3}+9 p_{3} \phi_{3,3}+16 p_{4} \phi_{3,4}=0 \\
\cdots \ldots \\
(n-2)^{2} p_{n-2} \phi_{n-2, n-1}+(n-1)^{2} p_{n-1} \phi_{n-1, n-1}+n^{2} p_{n} \phi_{n-1, n}=0 \\
p_{1} \phi_{1, n}+(n-1)^{2} p_{n-1} \phi_{n-1, n}+n^{2} p_{n} \phi_{n, n}=0 .
\end{array}\right.
$$

We rewrite this system as

$$
M \mathbf{x}=0,
$$

where the matrix $M$ (also denoted by $M=M_{n}$ ) is given by

$$
M=\left[\begin{array}{cccccccccccc}
p_{1} & 0 & 0 & \ldots & 0 & 0 & 2 p_{2} & 0 & 0 & \ldots & 0 & n p_{n} \\
0 & 2 p_{2} & 0 & \ldots & 0 & 0 & p_{1} & 3 p_{3} & 0 & \ldots & 0 & 0 \\
0 & 0 & 3 p_{3} & \ldots & 0 & 0 & 0 & 2 p_{2} & 4 p_{4} & \ldots & 0 & 0 \\
\ldots & \ldots & \ldots & \ldots & \ldots & \ldots & \ldots & \ldots & \ldots & \ldots & \ldots & \ldots \\
0 & 0 & 0 & \ldots & (n-1) p_{n-1} & 0 & 0 & 0 & \ldots & \ldots & n p_{n} & 0 \\
0 & 0 & 0 & \ldots & 0 & n p_{n} & 0 & 0 & \ldots & 0 & (n-1) p_{n-1} & p_{1} \\
p_{1} & 0 & 0 & \ldots & 0 & 0 & 4 p_{2} & 0 & 0 & 0 & 0 & n^{2} p_{n} \\
0 & 4 p_{2} & 0 & \ldots & 0 & 0 & p_{1} & 9 p_{3} & 0 & \ldots & 0 & 0 \\
0 & 0 & 9 p_{3} & \ldots & 0 & 0 & 0 & 4 p_{2} & 16 p_{4} & \ldots & 0 & 0 \\
\ldots & \ldots & \ldots & \ldots & \ldots & \ldots & \ldots & \ldots & \ldots & \ldots & \ldots & \ldots \\
0 & 0 & 0 & \ldots & (n-1)^{2} p_{n-1} & 0 & 0 & 0 & \ldots & \ldots & n^{2} p_{n} & 0 \\
0 & 0 & 0 & \ldots & 0 & n^{2} p_{n} & 0 & 0 & 0 & \ldots & (n-1)^{2} p_{n-1} & p_{1}
\end{array}\right],
$$

the unknown vector $\mathbf{x}$ is ordered as $\mathbf{x}=\left(\phi_{1,1}, \phi_{2,2}, \phi_{3,3}, \ldots, \phi_{n, n}, \phi_{1,2}, \phi_{2,3}, \phi_{3,4}, \ldots \phi_{n-1, n}, \phi_{1, n}\right)$, and 0 is the vector with all $2 n$ components equal to zero.

It can be seen that, if each $p_{h}$ (for $h=1, \ldots, n$ ) is different from zero, the matrix $M$ has determinant zero whereas its rank is equal to $2 n-1$. Below, we first work out the calculations for the case $n=4$, which is the smallest positive integer for which the particular structure of the matrix $\phi$ is clearly recognisable. Then, we describe the procedure to handle the case with general $n$. 
Denote by $M_{4}$ the matrix $M$ with $n=4$.

Proposition 1. The $8 \times 8$ matrix $M_{4}$ has determinant equal to zero and rank equal to seven.

Proof. The matrix $M_{4}$ has the form

$$
M_{4}=\left[\begin{array}{cccccccc}
p_{1} & 0 & 0 & 0 & 2 p_{2} & 0 & 0 & 4 p_{4} \\
0 & 2 p_{2} & 0 & 0 & p_{1} & 3 p_{3} & 0 & 0 \\
0 & 0 & 3 p_{3} & 0 & 0 & 2 p_{2} & 4 p_{4} & 0 \\
0 & 0 & 0 & 4 p_{4} & 0 & 0 & 3 p_{3} & p_{1} \\
p_{1} & 0 & 0 & 0 & 4 p_{2} & 0 & 0 & 16 p_{4} \\
0 & 4 p_{2} & 0 & 0 & p_{1} & 9 p_{3} & 0 & 0 \\
0 & 0 & 9 p_{3} & 0 & 0 & 4 p_{2} & 16 p_{4} & 0 \\
0 & 0 & 0 & 16 p_{4} & 0 & 0 & 9 p_{3} & p_{1}
\end{array}\right] .
$$

By substituting the 5-th row with that obtained as the difference of the 5-th row minus the first row, we get a matrix, whose determinant is easily seen to be equal to

$$
\operatorname{det}\left(M_{4}\right)=p_{1} \times \operatorname{det}\left(M_{4,\{7 \times 7\}}\right),
$$

where

$$
M_{4,\{7 \times 7\}}=\left[\begin{array}{ccccccc}
2 p_{2} & 0 & 0 & p_{1} & 3 p_{3} & 0 & 0 \\
0 & 3 p_{3} & 0 & 0 & 2 p_{2} & 4 p_{4} & 0 \\
0 & 0 & 4 p_{4} & 0 & 0 & 3 p_{3} & p_{1} \\
0 & 0 & 0 & 2 p_{2} & 0 & 0 & 12 p_{4} \\
4 p_{2} & 0 & 0 & p_{1} & 9 p_{3} & 0 & 0 \\
0 & 9 p_{3} & 0 & 0 & 4 p_{2} & 16 p_{4} & 0 \\
0 & 0 & 16 p_{4} & 0 & 0 & 9 p_{3} & p_{1}
\end{array}\right] .
$$

By substituting the 5-th row with that obtained as the difference of the 5-th row minus 2 times the first row, we get a matrix, whose determinant is easily seen to be equal to

$$
\operatorname{det}\left(M_{4,\{7 \times 7\}}\right)=2 p_{2} \times \operatorname{det}\left(M_{4,\{6 \times 6\}}\right),
$$

where

$$
M_{4,\{6 \times 6\}}=\left[\begin{array}{cccccc}
3 p_{3} & 0 & 0 & 2 p_{2} & 4 p_{4} & 0 \\
0 & 4 p_{4} & 0 & 0 & 3 p_{3} & p_{1} \\
0 & 0 & 2 p_{2} & 0 & 0 & 12 p_{4} \\
0 & 0 & -p_{1} & 3 p_{3} & 0 & 0 \\
9 p_{3} & 0 & 0 & 4 p_{2} & 16 p_{4} & 0 \\
0 & 16 p_{4} & 0 & 0 & 9 p_{3} & p_{1}
\end{array}\right] .
$$

With two similar further steps (i.e., iteratively suitably substituting the 5-th row of a matrix), one easily finds that

$$
\operatorname{det}\left(M_{4}\right)=p_{1} \times 2 p_{2} \times 3 p_{3} \times 4 p_{4} \times \operatorname{det}\left(M_{4,\{4 \times 4\}}\right),
$$

where

$$
M_{4,\{4 \times 4\}}=\left[\begin{array}{cccc}
2 p_{2} & 0 & 0 & 12 p_{4} \\
-p_{1} & 3 p_{3} & 0 & 0 \\
0 & -2 p_{2} & 4 p_{4} & 0 \\
0 & 0 & -3 p_{3} & -3 p_{1}
\end{array}\right]
$$

The Laplace expansion of $\operatorname{det}\left(M_{4,\{4 \times 4\}}\right)$, iteratively applied, gives

$$
2 p_{2}\left[\left(-3 p_{1}\right)\left(3 p_{3} 4 p_{4}\right)\right]+(-1) 12 p_{4}\left[\left(-p_{1}\right)\left(-2 p_{2}\right)\left(-3 p_{3}\right)\right]=0,
$$

which in turn implies that $\operatorname{det}\left(M_{4}\right)=0$.

In view of (31), in order to conclude that the rank of $M_{4}$ is equal to seven; it is sufficient to prove that $\operatorname{rank}\left(M_{4,\{4 \times 4\}}\right)=3$. And this can be immediately seen because, for example, the minor corresponding to the determinant of the triangular $3 \times 3$ matrix

$$
\left[\begin{array}{ccc}
2 p_{2} & 0 & 0 \\
-p_{1} & 3 p_{3} & 0 \\
0 & -2 p_{2} & 4 p_{4}
\end{array}\right]
$$

is different from zero (being $p_{j} \neq 0$ for all indices $j=1, \ldots, n$ by assumption).

The proof strategy can be generalised for the case of the $2 n \times 2 n$ matrix $M$ leading to the following result.

Proposition 2. For the $2 n \times 2 n$ matrix $M$, it is $\operatorname{det}(M)=0$ and $\operatorname{rank}(M)=2 n-1$.

Proof. By performing $n$ times a procedure similar to that in the proof of the previous proposition, namely,

(i) Step 1: substituting the $(n+1)$-th row of the matrix $M$ with that obtained as the difference of the $(n+1)$-th row minus the first row

(ii) Step 2: substituting the $(n+1)$-th row of the $(2 n-$ $1) \times(2 n-1)$ matrix $M_{(2 n-1) \times(2 n-1)}$ obtained after elimination of the first row and the first column from the matrix resulting from the previous step with that obtained as the difference of the $(n+1)$-th row minus 2 times the first row

(iii) ...

(iv) step $n$ : substituting the $(n+1)$-th row of the $(n+$ 1) $\times(n+1)$ matrix $M_{(n+1) \times(n+1)}$ obtained after elimination of the first row and the first column from the matrix resulting from the previous step 
with that obtained as the difference of the $(n+1)$-th row minus $n$ times the first row

one finds that

$$
\operatorname{det}(M)=p_{1} \times 2 p_{2} \times \cdots \times n p_{n} \times \operatorname{det}\left(M_{\{n \times n\}}\right),
$$

where

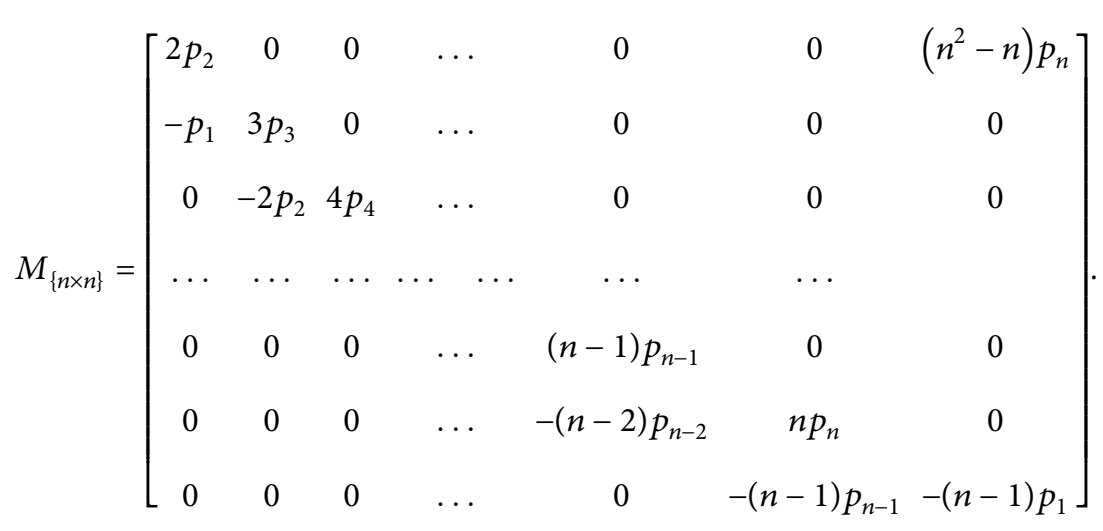

The determinant $\operatorname{det}\left(M_{\{n \times n\}}\right)$ can be calculated by iteratively applying the Laplace expansion. It is not difficult to convince oneself that

$$
\begin{aligned}
\operatorname{det}\left(M_{\{n \times n\}}\right) & =2 p_{2}^{3} p_{3}, \ldots, n p_{n}\left(-(n-1) p_{1}\right)+(-1)^{n+1}\left(n^{2}-n\right) p_{n}(-1)^{n+1}\left(p_{1}^{2} p_{2}, \ldots,(n-1) p_{n-1}\right) \\
& =\left(p_{1}^{2} p_{2}, \ldots,(n-1) p_{n-1}\right)\left(-n^{2} p_{n}+n p_{n}+n^{2} p_{n}-n p_{n}\right)=0 .
\end{aligned}
$$

Together, (35) and (37) imply that $\operatorname{det}(M)=0$.

It only remains to be proved that the rank of $M$ is equal to $2 n-1$. Also here, similarly as in the proof of Proposition 1 , we observe that the $(n-1) \times(n-1)$ matrix obtained by deleting the last row and the last column in $M_{\{n \times n\}}$ has determinant equal to $p_{1} 2 p_{2}, \ldots, n p_{n} \neq 0$. Hence, $\operatorname{rank}\left(M_{\{n \times n\}}\right)=n-1$ and, together with (35), this completes the proof.

Proposition 2 implies that the eigenspace of $M$ is onedimensional, and this in turn means that equation (24) admits infinitely many solutions; precisely, there is a oneparameter family of them. By way of example, let us consider the following low-dimensional case.

Example 1. Let $n=4$ and let a Markovian network $\mathcal{N}_{4}$ with degree distribution $P(h)$ and correlation matrix $P(h \mid k)$ be given. Assume that $p_{h}=P(h) \neq 0$ for $h=1, \ldots, n$. The function $k_{n n}$ pertaining to $\mathcal{N}_{4}$ is also the average nearest neighbor degree of the networks which have the same degree distribution $p_{h}$ as $\mathcal{N}_{4}$ and correlation matrix of the form $P(h \mid k)+h p_{h} /\langle k\rangle \phi_{h, k}$, the only nonzero elements of the symmetric matrix $\phi_{h, k}$ being

$$
\begin{aligned}
& \phi_{1,1}, \\
& \phi_{2,2}=\frac{3 p_{1}^{2}}{4 p_{2}^{2}} \phi_{1,1}, \\
& \phi_{3,3}=\frac{p_{1}^{2}}{3 p_{3}^{2}} \phi_{1,1}, \\
& \phi_{4,4}=\frac{p_{1}^{2}}{16 p_{4}^{2}} \phi_{1,1}, \\
& \phi_{1,2}=-\frac{3 p_{1}}{4 p_{2}} \phi_{1,1}, \\
& \phi_{2,3}=-\frac{p_{1}^{2}}{4 p_{2} p_{3}} \phi_{1,1}, \\
& \phi_{3,4}=-\frac{p_{1}^{2}}{8 p_{3} p_{4}} \phi_{1,1}, \\
& \phi_{1,4}=\frac{p_{1}}{8 p_{4}} \phi_{1,1},
\end{aligned}
$$


all of them expressed in terms of a unique parameter $\phi_{1,1}$, together with those symmetrically positioned with respect to the main diagonal, provided the inequalities

$$
\phi_{h, k} \geq-\frac{\langle k\rangle}{h p_{h}} P(h \mid k), \quad \text { for all } h, k=1, \ldots, n,
$$

are satisfied.

We recall here that $P(h \mid k) \geq 0$ for all $h, k=1, \ldots, n$. Therefore, the inequalities to be checked are in fact those relative to the nonzero elements $\phi_{h, k}$. In this example $(n=4)$, they can be expressed as

$$
\left\{\begin{array}{l}
\phi_{h, h} \geq-\frac{\langle j\rangle}{h p_{h}} P(h \mid h), \quad \text { for all } h=1, \ldots, 4, \\
\phi_{1,2} \geq \max \left\{-\frac{\langle j\rangle}{p_{1}} P(1 \mid 2),-\frac{\langle j\rangle}{2 p_{2}} P(2 \mid 1)\right\}, \\
\phi_{2,3} \geq \max \left\{-\frac{\langle j\rangle}{2 p_{2}} P(2 \mid 3),-\frac{\langle j\rangle}{3 p_{3}} P(3 \mid 2)\right\}, \\
\phi_{3,4} \geq \max \left\{-\frac{\langle j\rangle}{3 p_{3}} P(3 \mid 4),-\frac{\langle j\rangle}{4 p_{4}} P(4 \mid 3)\right\}, \\
\phi_{1,4} \geq \max \left\{-\frac{\langle j\rangle}{p_{1}} P(1 \mid 4),-\frac{\langle j\rangle}{4 p_{4}} P(4 \mid 1)\right\} .
\end{array}\right.
$$

Explicit treatment of an example requires fixing both the values of the elements of the degree distribution $p_{h}$ and correlation matrix $P(h \mid k)$. We here consider three cases, each of them relative to a scale-free network with $p_{h}=c / h^{\gamma}$, where $\gamma \in(2,3)$, and $P(h \mid k)$ is constructed according to the following algorithms (see $[12,19])$ :

(i) Case 1. We define $P_{0}(h \mid k)$ as follows: for $h<k$, $P_{0}(h \mid k)=|h-k|^{-1}$, for $h=k, P_{0}(h \mid k)=1$, and

$$
P_{0}(h \mid k)=P_{0}(k \mid h) \frac{h^{1-\gamma}}{k^{1-\gamma}}, \quad \text { if } h>k .
$$

Call $C_{k}=\sum_{h=1}^{n} P_{0}(h \mid k)$ for any $k=1, \ldots, n$ and let $C_{\text {max }}=\max _{k=1, \ldots, n} C_{k}$. Then, redefine the correlation matrix by setting the elements on the diagonal equal to

$$
P_{1}(k \mid k)=C_{\max }-C_{k}, \quad k=1, \ldots, n,
$$

and leaving the other elements unchanged: $P_{1}(h \mid k)=P_{0}(h \mid k)$ for $h \neq k$. Finally, normalize the entire matrix by setting

$$
P(h \mid k)=\frac{1}{\left(C_{\max }-1\right)} P_{1}(h \mid k), \quad h, k=1, \ldots, n .
$$

(ii) Case 2. Let $P_{0}(h \mid k)=1-1 / N|h-k|$ if $h \leq k$ and $P_{0}(h \mid k)$ with $h>k$ as in (41). Then, proceed as in the previous case to get elements $P(h \mid k)$ which satisfy the normalization $\sum_{h=1}^{n} P(h \mid k)=1$.

(iii) Case 3. Let $P_{0}(h \mid k)=e^{-(h-k)^{2} / n^{2}}$ if $h \leq k$ and $P_{0}(h \mid k)$ with $h>k$ as in (41). Again, proceed as above to get elements $P(h \mid k)$ which satisfy the normalization $\sum_{h=1}^{n} P(h \mid k)=1$.

Straightforward calculations (performed with Mathematica) yield, for example, that inequalities (40) are satisfied in the following situations:

In Case 1 , with $\gamma=2.1$, provided $0 \leq \phi_{1,1} \leq 0.12908$ holds true

In Case 1 , with $\gamma=2.5$, provided $0 \leq \phi_{1,1} \leq 0.06478$ holds true

In Case 1, with $\gamma=2.9$, provided $0 \leq \phi_{1,1} \leq 0.03369$ holds true

In Case 2, with $\gamma=2.1$, provided $0 \leq \phi_{1,1} \leq 0.12010$ holds true

In Case 2, with $\gamma=2.5$, provided $0 \leq \phi_{1,1} \leq 0.06012$ holds true

In Case 2, with $\gamma=2.9$, provided $0 \leq \phi_{1,1} \leq 0.03119$ holds true

In Case 3, with $\gamma=2.1$, provided $0 \leq \phi_{1,1} \leq 0.11247$ holds true

In Case 3, with $\gamma=2.5$, provided $0 \leq \phi_{1,1} \leq 0.05618$ holds true

In Case 3, with $\gamma=2.9$, provided $0 \leq \phi_{1,1} \leq 0.02876$ holds true

When calculating the connectivity matrix $C_{k, h}$ in correspondence to correlations $P(h \mid k)$ and then also in correspondence to correlations $P(h \mid k)+\Phi(h, k)$ in the Cases 1,2, and 3 above, for $\gamma=2.1,2.5,2.9$ and (various) values of $\phi_{1,1}$ compatible with the intervals just found, one observes what follows. In passing from $P(h \mid k)$ to $P(h \mid k)+\Phi(h, k)$ (namely, by taking an admissibile positive $\phi_{1,1}$ rather than $\left.\phi_{1,1}=0\right)$, in all Cases, 1,2 , and 3 , the largest eigenvalue of $C_{k, h}$ increases (and, accordingly, the epidemic threshold decreases).

In contrast, if one takes an uncorrelated network, by this meaning a network for which

(iv) Case 4. $P(h \mid k)=h P(h) /\langle k\rangle$,

and considers then the matrix $P(h \mid k)+\Phi(h, k)$ with elements $\Phi(h, k)$ constructed according to (20) and (22), one notices the following fact: the largest eigenvalue of the connectivity matrix $C_{k, h}$ remains equal to $\left\langle k^{2}\right\rangle /\langle k\rangle$ when the parameter $\phi_{1,1}$ varies in the interval which guarantees the meaningfulness of the variation $\left(-0.01234<\phi_{1,1}<0.01603\right)$. A subtle and interesting situation is taking place: on the one hand, for networks constructed considering the elements $P(h \mid k)+\Phi(h, k)$ as done here, it is no more true that the conditional probability that a vertex of degree $k$ is connected to a vertex of degree $h$ which is independent of $k$; on the other hand, the average nearest neighbor degree function $k_{n n}(k)$ of these networks is the same of that of a strictly 
uncorrelated network; it is constant (and equal to $\left\langle k^{2}\right\rangle /\langle k\rangle$, coinciding with $k_{n n}(k)$ of the original uncorrelated network).

Remark 1. Beside the choice of taking symmetric matrices $\phi$ as in (22), other choices can be performed. They lead both to cases in which the largest eigenvalue of the connectivity matrix in correspondence to the matrix $P(h \mid k)+\Phi(h, k)$ is greater than the one obtained in correspondence to the matrix $P(h \mid k)$ as to cases in which this eigenvalue is smaller.

In any case, one can conclude that networks with the same $k_{n n}$ but different correlation matrices can exhibit different epidemic thresholds.

\section{Data Availability}

No data were used to support this study.

\section{Conflicts of Interest}

The authors declare that they have no conflicts of interest.

\section{Acknowledgments}

This work was supported by the Open Access Publishing Fund of the Free University of Bozen-Bolzano.

\section{References}

[1] S. Boccaletti, V. Latora, Y. Moreno, M. Chavez, and D.-U. Hwang, "Complex networks: structure and dynamics," Physics Reports, vol. 424, no. 4-5, pp. 175-308, 2006.

[2] M. E. J. Newman, Networks: An Introduction, Oxford University Press, Oxford, UK, 2010.

[3] A.-L. Barabási, Network Science, Cambridge University Press, Cambridge, UK, 2016.

[4] M. Boguñá, R. Pastor-Satorras, and A. Vespignani, "Epidemic spreading in complex networks with degree correlations," in Statistical Mechanics of Complex NetworksSpringer, Heidelberg, Germany, 2003.

[5] M. L. Bertotti and G. Modanese, "The configuration model for Barabasi-Albert networks," Applied Network Science, vol. 4, no. 1, p. 32, 2019.

[6] R. Pastor-Satorras, C. Castellano, P. Van Mieghem, and A. Vespignani, "Epidemic processes in complex networks," Reviews of Modern Physics, vol. 87, no. 3, pp. 925-979, 2015.

[7] M. Boguñá, R. Pastor-Satorras, and A. Vespignani, "Absence of epidemic threshold in scale-free networks with degree correlations," Physical Review Letters, vol. 90, no. 2, Article ID 028701, 2003.

[8] M. Boguñá and R. Pastor-Satorras, "Epidemic spreading in correlated complex networks," Physical Review E, vol. 66, no. 4, Article ID 047104, 2002.

[9] M. E. Newman, "Mixing patterns in networks," Physical Review E, vol. 67, no. 2, Article ID 026126, 2003.

[10] R. Noldus and P. Van Mieghem, "Assortativity in complex networks," Journal of Complex Networks, vol. 3, no. 4, pp. 507-542, 2015.

[11] M. L. Bertotti and G. Modanese, "Network rewiring in the $\mathrm{r}-\mathrm{K}$ plane," Entropy, vol. 22, no. 6, p. 653, 2020.
[12] M. L. Bertotti and G. Modanese, "Comparison of simulations with a mean-field approach vs. synthetic correlated networks," Symmetry, vol. 13, no. 1, p. 141, 2021.

[13] S. Weber and M. Porto, "Generation of arbitrarily two-pointcorrelated random networks," Physical Review E, vol. 76, no. 4, Article ID 046111, 2007.

[14] D. H. Silva, S. C. Ferreira, W. Cota, R. Pastor-Satorras, and C. Castellano, "Spectral properties and the accuracy of meanfield approaches for epidemics on correlated power-law networks," Physical Review Research, vol. 1, no. 3, Article ID 033024, 2019.

[15] A. Vázquez and M. Weigt, "Computational complexity arising from degree correlations in networks," Physical Review E, vol. 67, no. 2, Article ID 027101, 2003.

[16] M. Nekovee, Y. Moreno, G. Bianconi, and M. Marsili, "Theory of rumour spreading in complex social networks," Physica A: Statistical Mechanics and its Applications, vol. 374, no. 1, pp. 457-470, 2007.

[17] A. Vázquez and Y. Moreno, "Resilience to damage of graphs with degree correlations," Physical Review E, vol. 67, no. 1, Article ID 015101, 2003.

[18] S. N. Dorogovtsev and J. F. F. Mendes, "Evolution of networks," Advances in Physics, vol. 51, no. 4, pp. 1079-1187, 2002.

[19] M. L. Bertotti and G. Modanese, "On the evaluation of the takeoff time and of the peak time for innovation diffusion on assortative networks," Mathematical and Computer Modelling of Dynamical Systems, vol. 25, no. 5, pp. 482-498, 2019. 